\title{
L'enquête Enveff-Martinique
}

Méthodologie et composition de l'échantillon

The Enveff-Martinique survey: methodology and sampling

\section{Elizabeth Brown et Nadine Lefaucheur}

\section{(2) OpenEdition}

Journals

Édition électronique

URL : http://journals.openedition.org/plc/862

DOI : $10.4000 /$ plc. 862

ISSN : 2117-5209

Éditeur

L'Harmattan

Édition imprimée

Date de publication : 1 janvier 2012

Pagination : 63-71

ISBN : 978-2-296-55-856-4

ISSN : $1279-8657$

Référence électronique

Elizabeth Brown et Nadine Lefaucheur, "L'enquête Enveff-Martinique », Pouvoirs dans la Caraïbe [En ligne], 17 | 2012, mis en ligne le 31 janvier 2012, consulté le 19 avril 2019. URL : http://

journals.openedition.org/plc/862 ; DOI : 10.4000/plc.862 


\title{
L'ENQUETTE ENVEFF-MARTINIQUE MÉTHODOLOGIE ET COMPOSITION DE L'ÉCHANTILLON
}

\author{
Elizabeth BROWN \\ Directrice du Centre de recherche de \\ l'Institut de démographie \\ Université Paris1 Panthéon-Sorbonne
}

\section{Nadine LEFAUCHEUR}

Sociologue

Membre associé du CRPLC

Université des Antilles et de la Guyane

L'enquête statistique Genre et violences interpersonnelles en Martinique s'inscrit dans la ligne des enquêtes Enveff, dont elle reprend la problématique, les principes et la méthodologie. Comme les précédentes, l'enquête réalisée en Martinique n'a pas porté sur la seule violence conjugale, mais envisagé tous les espaces de vie où les répondant(e)s ont pu subir des violences, et n'a pas non plus présupposé que les violences subies par les femmes aient toujours été le fait d'hommes ni que les femmes aient toujours été victimes et jamais auteurs de violences ${ }^{1}$. D'ailleurs, à titre expérimental, on s'est adressé aux hommes et aux femmes à l'aide d'un même questionnaire, dans le but de mesurer la fréquence des violences verbales, psychologiques, physiques et sexuelles que les uns et les autres ont pu subir dans l'espace public, au travail, dans le cadre des relations conjugales ou dans l'environnement familial et amical. Les travaux sur la socialisation masculine montrent en effet que l'inculcation d'un ethos et d'un habitus de domination se fait largement à travers des insultes, des violences psychologiques et physiques, qui éprouvent la capacité tant à endurer la violence qu'à l'exercer contre autrui. Toutefois, postuler que les hommes aussi sont victimes de violences, n'implique pas de considérer qu'ils le sont comme les femmes. Outre le sexe des auteurs des faits violents déclarés et les différences entre les sexes dans les réactions et les conséquences que ces faits ont entraînées, il s'agissait donc d'identifier la dangerosité différentielle des espaces de vie et de relations, ainsi que des âges de la vie, pour les hommes et pour les femmes.

\footnotetext{
${ }^{1}$ Selon l'Observatoire national de la délinquance et des réponses pénales, les hommes représentent un sixième des victimes de violences volontaires par conjoint constatées en zones gendarmerie, essentiellement rurales (INHES/OND, rapport 2008, p. 304). Notons cependant qu'il peut s'agir de violences à sens unique, dont l'homme est seul victime, ou, plus souvent, de violences réciproques, lors desquelles l'homme peut avoir frappé le premier.
} 
A la suite de l'enquête pilote réalisée en juin 2007, le recueil des données a été effectué en octobre-novembre 2008, selon la technique Cati (collecte assistée par téléphone et informatique), que nous avons estimée être la plus apte à assurer la confidentialité dans un espace insulaire où « tout le monde se connaît » et à faciliter un questionnement sur un sujet sensible, qui touche à l'intimité des personnes. Le fait que l'institut de sondage, choisi à la suite d'un appel d'offres ${ }^{2}$, ait été basé dans la région parisienne entraînait certes l'inconvénient du décalage horaire entre l'hexagone et les Antilles. Mais, l'accent immédiatement perçu comme «non antillais» de ses enquêteurs était apparu, dès l'enquête pilote, comme un gage supplémentaire de confidentialité assurant les répondant(e)s que leurs interlocuteurs ne pouvaient pas les connaître personnellement et risquer de makréler ou kankanner sur leur compte.

Le questionnaire, inspiré de celui qui a été utilisé dans l'hexagone en 2000, mais assez largement modifié pour s'adapter aux réalités locales, est structuré en sept modules. Le premier porte sur les caractéristiques familiales, économiques, sociales et résidentielles des répondant(e)s, de leurs parents et de leurs conjoints ou partenaires, lorsqu'ils/elles en ont (et, dans ce cas, sur l'entente conjugale au quotidien), ainsi que sur des éléments de leur parcours biographique. Ces informations sont destinées à tenter de mieux appréhender l'origine et le contexte d'apparition des faits de violence subis.

Les cinq modules suivants sont consacrés aux violences verbales, psychologiques, physiques et sexuelles subies pendant les douze derniers mois :

- dans la sphère professionnelle ou des études ;

- dans la vie conjugale et amoureuse ;

- dans une éventuelle relation avec un ancien conjoint ou avec un partenaire secondaire ;

- dans les relations familiales (non conjugales) ou avec les voisins, les amis et les proches ;

- dans les espaces et lieux publics.

Le dernier module est consacré aux principales agressions et violences, particulièrement physiques et sexuelles, subies avant et/ou après l'âge de 18 ans (à l'exception des douze derniers mois).

\footnotetext{
${ }^{2}$ Le montant du devis, mais aussi l'expérience d'enquêtes sur de tels sujets et la réalisation antérieure de plusieurs enquêtes nationales ou départementales Enveff ont déterminé ce choix.
} 
Dans l'objectif d'inclure dans l'étude la fraction de la population ne possédant pas de ligne fixe, un questionnaire court a été mis au point pour l'interrogation sur téléphone mobile ${ }^{3}$. Il comprend deux parties : la première reprend les principales questions du premier module du questionnaire long ; la seconde reprend le dernier module de ce questionnaire, en y incluant les faits subis au cours des douze derniers mois.

Afin de ne pas préjuger de ce qui est ou non considéré par les répondant(e)s comme de la violence, l'enquête a été, à l'instar des précédentes, présentée comme une enquête sur la santé et la sécurité ; l'emploi des termes «violent» et «violence» a été exclu au profit de l'énoncé, pour chaque espace, de faits et d'actes précis (insultes, menaces et agressions verbales, chantage ou pressions psychologiques, agressions physiques, pratiques et relations sexuelles imposées) dont les personnes enquêtées auraient pu être victimes.

Les échantillons de numéros téléphoniques ont été constitués au hasard selon deux procédés : pour les lignes fixes, les numéros ont été tirés au hasard sur la liste d'abonnés fournie par France Télécom, et incrémentés pour permettre l'inclusion de numéros d'abonnés sur liste rouge et d'abonnés d'Outremer Télécom; les numéros de mobiles ont été obtenus en générant au hasard les six chiffres à accoler à l'indicatif 0696 (mobiles Martinique). Des quotas d'interrogation proportionnels à leur population ont été établis pour les six régions: Fort-de-France/Lamentin, Sud caraïbe, Centre atlantique, Sud atlantique, Nord caraïbe et Nord atlantique.

Le questionnaire long a été administré à 1000 femmes et à 500 hommes âgés de 18 à 59 ans, tandis que le questionnaire court a été proposé à 152 femmes, contactées sur ligne mobile (56 n'avaient pas de ligne fixe) et à 454 hommes, contactés en partie sur ligne fixe et en partie sur ligne mobile (192 ne disposaient pas de ligne fixe) $)^{4}$. De ce fait, un certain nombre de données concernant les hommes portent seulement sur ceux qui ont répondu au questionnaire long, et ont une moindre significativité statistique que les données correspondantes concernant les femmes ${ }^{5}$.

\footnotetext{
${ }^{3}$ En 2006, 81,6\% des ménages martiniquais disposaient d'une ligne fixe et 65,5\% d'une ligne mobile. Parmi les $18,4 \%$ qui n'avaient pas de ligne fixe, $13,6 \%$ avaient une (ou plusieurs) lignes mobiles et $4,8 \%$ n'avaient ni ligne fixe ni ligne mobile (Sources : Enquêtes Budget des familles, Insee, et Conditions de vie et aspirations des ménages, Credoc).

${ }^{4}$ L'objectif initial était d'interroger 1000 femmes et 1000 hommes âgés de 18 à 69 ans avec le questionnaire long, plus 200 femmes et 200 hommes joints sur leur mobile avec le questionnaire court. Des contraintes à la fois techniques et financières ont conduit à ramener l'âge maximal à 59 ans et à reporter une partie de l'échantillon masculin sur l'enquête courte.

${ }^{5}$ Les personnes interrogées sur ligne mobile qui n'avaient pas de ligne fixe étaient en moyenne plus jeunes que les autres, vivaient moins souvent en couple, étaient plus souvent
} 


\section{I. - LA COMPOSITION DE L'ÉCHANTILLON}

Parmi l'ensemble des répondant(e)s ${ }^{6}, 65 \%$ des hommes et $51,3 \%$ des femmes étaient des actifs occupés, $14,6 \%$ des premiers et $19,3 \%$ des secondes se déclaraient chômeurs, et respectivement $7,1 \%$ et $12,7 \%$ retraités; un peu plus de $9 \%$ des hommes et des femmes se disaient étudiants; il reste donc $4,2 \%$ d'inactifs et $7,1 \%$ d'inactives. $12 \%$ des hommes et $20 \%$ des femmes qui travaillaient au moment de l'enquête ont également fait des études ou suivi une formation au cours des douze derniers mois. De même, un étudiant sur cinq et près d'une étudiante sur cinq ont dit travailler parallèlement ou avoir auparavant occupé un emploi ou fait des djobs $^{7}$.

Parmi les actifs occupés, $22 \%$ des hommes et $10 \%$ des femmes étaient des non-salariés (agriculteurs, pêcheurs, commerçants, professions libérales, chefs d'entreprise) ; $23 \%$ des hommes et $32 \%$ des femmes se sont déclarés fonctionnaires (plus souvent dans la fonction publique d'Etat ou territoriale pour les hommes, dans l'éducation nationale pour les femmes); $41 \%$ des hommes et $36 \%$ des femmes étaient des salariés non fonctionnaires en CDI ; $9 \%$ des hommes et $18 \%$ des femmes étaient en $\mathrm{CDD}$, période d'essai ou stage rémunéré ; $5 \%$ des hommes et $4 \%$ des femmes avaient un emploi aidé ou se sont déclarés djobeurs.

Les femmes étaient un peu plus diplômées que les hommes : moins d'un quart des premières et près de $30 \%$ des seconds n'avaient aucun diplôme ou seulement un CEP. Pour les niveaux intermédiaires, les deux sexes étaient à égalité : un tiers possédaient un BEPC, un CAP ou un BEP, et $16 \%$ un baccalauréat. Mais $28 \%$ des femmes et $21 \%$ des hommes étaient titulaires d'un diplôme d'études supérieures. Les actifs en emploi étaient proportionnellement plus nombreux à détenir une formation supérieure, avec

RMIstes ou chômeuses ; mais il y avait aussi plus souvent parmi elles à la fois des personnes sans diplôme et des personnes ayant un diplôme supérieur au baccalauréat.

${ }^{6}$ Les appels sur téléphone mobile ont permis de compenser en partie les distorsions de l'échantillon interrogé sur ligne fixe qui, comme dans la plupart des enquêtes où les personnes sont sollicitées à leur domicile, présentait un déficit de répondants appartenant aux groupes d'âges où les taux d'activité sont les plus élevés. Néanmoins, la distribution par âge de l'échantillon global, issu des deux séries de questionnaires, n'était pas parfaitement représentative de la structure de la population âgée de 18 à 59 ans vivant en Martinique. Des coefficients de calage, basés sur les données estimées par l'INSEE pour le $1^{\mathrm{er}}$ janvier 2008 (Estimation de population au $1^{\text {er }}$ janvier, par région, sexe et âge quinquennal, ElpReg_quin90-08.xls), ont donc été calculés pour chaque sexe et par groupe quinquennal d'âges et appliqués systématiquement de sorte que les résultats (tous «redressés ») présentés ici sont comparables à ceux qui émanent d'autres sources.

7 «Petits boulots », généralement non déclarés et réalisés à la demande pour divers «employeurs». 
le même écart entre les sexes, en faveur des femmes (quatre femmes sur dix contre trois hommes sur dix).

Les indicateurs de revenu et de niveau de vie ont, au contraire des indicateurs de diplômes, montré une situation plus favorable pour les hommes que pour les femmes, ce qui n'a pas constitué une grande surprise. Trois hommes sur quatre et un peu plus de huit femmes sur dix ont déclaré ne pas avoir de revenu personnel ou avoir un revenu mensuel inférieur à $2000 €^{8}$. Les personnes nées en Martinique et qui y ont toujours vécu ont déclaré des revenus inférieurs à celles qui ont vécu au moins un an ailleurs : neuf sur dix des premières et sept sur dix des secondes ont indiqué percevoir moins de $2000 €$ par mois. Mais un quart des personnes nées hors de Martinique d'au moins un parent né dans la Caraïbe et $45 \%$ des personnes nées en France métropolitaine de deux parents également nés dans l'hexagone se situaient dans les niveaux supérieurs à $2000 €$. Par ailleurs, $38 \%$ des femmes et $30 \%$ des hommes ne disposaient pas de véhicule personnel et, parmi eux, $45 \%$ des hommes et $60 \%$ des femmes n'avaient pas le permis de conduire. Un quart des hommes, mais un tiers des femmes, ont dit bénéficier de la CMU.

Les deux tiers des enquêtés $(69,6 \%$ des hommes et $63,5 \%$ des femmes) habitaient une maison individuelle et la plupart en étaient propriétaires (60\% des hommes et $52 \%$ des femmes). Inversement, ceux et celles qui habitaient un appartement ou un studio (30\% des hommes, $36 \%$ des femmes) en étaient rarement propriétaires (moins d'un homme sur deux, une femme sur quatre).

Pour tenir compte de la complexité du peuplement de la Martinique et de l'influence potentielle de cette complexité et des itinéraires migratoires sur les situations et les comportements, en particulier en matière de violences subies, nous avons construit des indicateurs d'origine et de parcours migratoire des personnes interrogées, à partir des réponses portant sur leur lieu de naissance et sur celui de leurs parents, sur la durée et l'ancienneté de leurs séjours éventuels hors de Martinique, pour les natifs du département, ou sur l'ancienneté de leur arrivée sur le territoire, pour les non natifs. La très grande majorité des enquêtés - $81 \%$ des femmes et $84 \%$ des hommes sont nés en Martinique de parents eux-mêmes nés en Martinique. Sur cent personnes natives du département, 60 femmes et 54 hommes y ont toujours vécu, tandis que 19 femmes et 21 hommes ont vécu plus de cinq ans hors de

\footnotetext{
${ }^{8}$ Des tranches plus précises étaient proposées aux personnes interrogées au moyen du questionnaire long : plus de la moitié des femmes ont alors déclaré ne pas avoir de revenu personnel (11\%) ou n'avoir qu'un revenu inférieur à $1000 €$ par mois $(41 \%)$, ce qui n'était le cas que de quatre hommes sur dix ; $20 \%$ des hommes et $15 \%$ des femmes ont déclaré un revenu mensuel supérieur à $2000 €$ et $5 \%$ des hommes et $3 \%$ des femmes ont indiqué dépasser les $3500 €$ par mois.
} 
Martinique, le plus souvent en France hexagonale, le plus souvent après 18 ans et le plus souvent pour des raisons liées aux études. Plus des deux tiers des personnes qui ont vécu hors de Martinique (souvent âgées de plus de 40 ans) y sont revenues depuis plus de dix ans. Au total, $48 \%$ des enquêtés sont nés en Martinique et n'ont jamais vécu ailleurs, $17 \%$ sont nés en Martinique et ont vécu moins de cinq ans hors du département, $17 \%$ sont nés en Martinique et ont vécu plus de cinq ans hors du territoire, $6 \%$ sont nées dans l'hexagone d'au moins un parent natif des Antilles, $6 \%$ sont nés dans l'hexagone de parents non natifs des Antilles, 3,4\% sont nés dans un autre pays de la Caraïbe et 2,6\% sont nés ailleurs.

$45 \%$ des hommes et $56 \%$ des femmes se sont déclarés catholiques, $45 \%$ des hommes et $31 \%$ des femmes se sont dits sans religion, tandis que $8 \%$ des hommes et $11 \%$ des femmes se sont réclamés de confessions issues du protestantisme (adventistes, évangélistes, témoins de Jéhovah) et que $2 \%$ des hommes comme des femmes ont indiqué d'autres religions (bouddhisme, rastafarisme, islam, orthodoxes, etc.).

\section{II. - LES RELATIONS CONJUGALES ET FAMILIALES}

Parmi les personnes enquêtées, $60 \%$ sont célibataires, $30 \%$ mariées et un peu plus de $5 \%$ divorcées, les autres étant séparées légalement, veuves ou pacsées. Jusqu'à 40 ans, la proportion de célibataires dépasse $75 \%$ pour les deux sexes. Ces résultats, qui portent sur les 18-59 ans, sont cohérents avec les données de l'INSEE qui indiquaient, pour l'année 2006, 55,8\% de célibataires, $34,3 \%$ de mariés, $4,9 \%$ de divorcées et $4,9 \%$ de veufs dans l'ensemble de la population martiniquaise âgée de 15 ans et plus.

Indépendamment du statut matrimonial, les enquêtes Enveff entendent la notion de relation conjugale au sens large, incluant toutes les personnes ayant répondu avoir, au moment de l'enquête, «une relation affective et/ou sexuelle suivie» (soit $69 \%$ des hommes et $66 \%$ des femmes).

Un peu plus de $40 \%$ des répondant(e)s (44\% des hommes et $41 \%$ des femmes, ayant souvent plus de 35 ans), ont déclaré vivre avec un conjoint : les deux tiers sont mariés et les trois quarts ont au moins un enfant présent au foyer. Dans un cas sur trois, le couple a des enfants de plusieurs lits ou d'un autre partenaire.

Par ailleurs, un peu plus de $35 \%$ des personnes ayant déclaré une relation en cours ont aussi dit ne pas vivre avec leur partenaire. Parmi ces personnes (plus souvent âgées de moins de 35 ans), la très grande majorité n'ont jamais été mariées et six hommes, mais seulement cinq femmes, sur dix n'ont aucun enfant. $7 \%$ des hommes, mais $43 \%$ des femmes en « couple 
non cohabitant» sont en fait chef de foyer monoparental. Pour une femme sur deux, le partenaire est le père d'au moins un de ses enfants.

Une personne sur cinq (19\% des hommes et $20 \%$ des femmes) a déclaré ne pas avoir de relation conjugale au moment de l'enquête, mais avoir déjà eu une relation de couple (avec ou sans cohabitation). Parmi ces personnes, $13 \%$ des hommes, mais les deux tiers des femmes, ont au moins un enfant présent au foyer.

Enfin, parmi les $13 \%$ de personnes qui ont répondu ne jamais avoir eu de relation de couple suivie, près de la moitié des femmes ont au moins un enfant.

Au total, parmi les personnes interrogées, $62 \%$ des femmes et $36 \%$ des hommes ont au moins un enfant présent au foyer ; $30 \%$ des femmes (33\% des natives), mais seulement $4 \%$ des hommes, sont en situation monoparentale. Le conjoint ou partenaire d'une femme sur trois de celles qui ont déclaré avoir une relation de couple au moment de l'enquête avait eu des enfants avec d'autres femmes, soit avant, soit pendant leur relation.

On retrouve cette même complexité des structures familiales dans l'enfance et l'adolescence de nombre de personnes enquêtées. Lorsqu'elles avaient quatorze ans, un peu moins des deux tiers des personnes enquêtées vivaient avec leurs deux parents, un quart avec leur mère, elle-même sans conjoint, $4 \%$ avec leur mère et un beau-père, $3 \%$ avec leur père seul ou avec leur père et une belle-mère et $5 \%$ avec leurs grands-parents ou un autre membre de la famille. Un peu plus de $20 \%$ avaient des parents séparés ou divorcés, $10 \%$ environ avaient des parents qui n'avaient jamais vécu ensemble, $5 \%$ environ étaient orphelins de père et/ou de mère et $2 \%$ de père inconnu.

La situation varie cependant selon l'âge des répondants : si $68 \%$ des plus de 40 ans vivaient avec leurs deux parents lorsqu'ils avaient quatorze ans, ce n'était le cas que de $60 \%$ des hommes et $54 \%$ des femmes de moins de 40 ans. Ainsi, la proportion des personnes dont les parents s'étaient séparés après avoir vécu ensemble passe de 13-14 \% pour les plus de 40 ans à $25-28 \%$ pour les moins de 40 ans.

Parmi les personnes enquêtées, $15 \%$ à $18 \%$ n'ont pas de frères et sœurs (de même père et même mère), mais un tiers en ont au moins quatre et $5 \%$ au moins dix ; $42 \%$ n'ont pas (à leur connaissance) de demi-frères ou sœurs, mais $28 \%$ au moins en ont du côté paternel seulement, $8 \%$ au moins en ont du côté maternel seulement et $19 \%$ des deux côtés. La moitié d'entre eux ont vécu avec au moins l'un de ces demi-frères ou sœurs - il s'agit le plus souvent, dans ce cas, de demi-frères ou sœurs utérins.

La composition de la fratrie des personnes qui, à quatorze ans, vivaient avec leurs deux parents, est proche de la moyenne des enquêtés. 
Mais, parmi celles qui vivaient avec leur mère seule, moins d'une sur cinq seulement n'a pas de demi-frères ou demi-sœurs, un tiers environ en a seulement du côté paternel, $12 \%$ du côté maternel seulement et un tiers environ des deux côtés. Parmi celles qui vivaient avec leur mère et un beaupère, $12 \%$ seulement n'ont pas de demi-frères ou de demi-sœurs : $18 \%$ au moins en ont du côté paternel seulement, $12 \%$ du côté maternel seulement et $55 \%$ au moins des deux côtés.

Les personnes qui, à quatorze ans, vivaient avec leur mère seule avaient moins souvent une relation de couple au moment de l'enquête que celles qui vivaient avec leurs deux parents et, surtout, vivaient deux fois moins fréquemment avec leur partenaire. 


\section{RÉSUMÉ}

Elizabeth Brown et Nadine Lefaucheur. L'enquête Enveff-Martinique : méthodologie et composition de l'échantillon

L'enquête Enveff-Martinique, réalisée dans le cadre du programme «Genre et violences interpersonnelles à la Martinique », a également porté, à titre exploratoire, sur les violences déclarées par les hommes ; 1152 femmes et 954 hommes, sélectionnés aléatoirement, ont été interrogés par téléphone ; 1000 femmes et 500 hommes l'ont été au moyen d'un questionnaire « long » comprenant sept modules (caractéristiques socio-démographiques ; violences subies au cours des douze derniers mois dans la sphère professionnelle, la vie conjugale ou amoureuse, les relations avec un ou une ex-partenaire, les relations avec la famille et les proches, les espaces et lieux publics ; violences subies antérieurement aux douze derniers mois) ; les autres ont fait l'objet d'un questionnement « court » limité à deux modules (socio-démographique ; violences au cours de la vie). Les réponses aux modules socio-démographiques fournissent de nombreuses données sur la société martiniquaise, en particulier pour ce qui concerne les parcours migratoires et les structures familiales.

\section{ABSTRACT \\ Elizabeth Brown and Nadine. LefaucheurThe Enveff-Martinique survey: methodology and sampling}

The Enveff-Martinique survey, conducted as part of the research program on "Gender and interpersonal violence in Martinique", also documented in an exploratory fashion the violence reported by men: 1,152 women and 954 men, randomly selected, were interviewed by telephone; 1,000 women and 500 men completed a long-form questionnaire consisting of seven modules (socio-demographic characteristics, violence experienced during the last twelve months in the professional sphere, in various, including public spaces, within romantic relationships with a current or ex-partner, within relationships with family and friends; and violence suffered prior to the last twelve months), others completed a short-form survey limited to two modules (socio-demographic questions and questions about violence throughout the life-cycle). Responses to the socio-demographic modules provide extensive data on Martinican society, particularly with regard to migration patterns and family structures. 